Roche, UCB, Novartis, Pfizer, Amgen, Consultant for: MSD, Abbvie, Roche, UCB, Novartis, Pfizer, Amgen, Speakers bureau: Pfizer, Onay Gercik: None declared, Duygu Ersözlü: None declared, Veli Yazısız: None declared, Gezmiş Kimyon: None declared, Müge Aydın: None declared, Rıdvan Mercan: None declared, Burak Öz: None declared, Nazife Sule Yasar Bilge: None declared, Zeynel Abidin Akar: None declared, Omer Karadag: None declared, Ayse Bahar Kelesoglu Dincer: None declared, Sedat Yilmaz: None declared, Ufuk İlgen: None declared, Yavuz Pehlivan: None declared, Ender Terzioğlu: None declared, Levent Kılıç: None declared, Şükran Erten: None declared, Sedat Kiraz: None declared DOI: 10.1136/annrheumdis-2019-eular.5279

\section{FRI0099 PREDICTIVE FACTORS FOR REMISSION ACHIEVEMENT BY TOCILIZUMAB MONOTHERAPY IN PATIENTS WITH RHEUMATOID ARTHRITIS AFTER INADEQUATE RESPONSE TO METHOTREXATE: A POST HOC ANALYSIS OF THE SURPRISE STUDY}

Masaru Kato ${ }^{1}$, Yuko Kaneko ${ }^{2}$, Yoshiya Tanaka ${ }^{3}$, Masayuki Inoo ${ }^{4}$, Hitomi Kobayashi-Haraoka ${ }^{5}$, Koichi Amano ${ }^{6}$, Masayuki Miyata ${ }^{7}$, Yohko Murakawa ${ }^{8}$, Hidekata Yasuoka ${ }^{2}$, Shintaro Hirata ${ }^{9}$, Hayato Nagasawa ${ }^{6}$, Eiichi Tanaka ${ }^{10}$, Nobuyuki Miyasaka $^{11}$, Hisashi Yamanaka ${ }^{10}$,

Kazuhiko Yamamoto' ${ }^{12}$, Isao Yokota ${ }^{13}$, Tatsuya Atsumi ${ }^{1}$, Tsutomu Takeuchi ${ }^{2}$. ${ }^{1}$ Hokkaido University, Department of Rheumatology, Endocrinology and Nephrology, Sapporo, Japan; ${ }^{2}$ Keio University School of Medicine, Tokyo, Japan; ${ }^{3}$ University of Occupational and Environmental Health, Kitakyushu, Japan; ${ }^{4}$ Utazu Hospital, Ehime, Japan; ${ }^{5}$ Nihon University School of Medicine, Tokyo, Japan; ${ }^{6}$ Saitama Medical University, Kawagoe, Japan; ${ }^{7}$ Fukushima Red Cross Hospital, Fukushima, Japan; ${ }^{8}$ Shimane University Faculty of Medicine, Izumo, Japan; ${ }^{9}$ Hiroshima University Hospital, Hiroshima, Japan; ${ }^{10}$ Tokyo Women's Medical University, Tokyo, Japan; ${ }^{11}$ Tokyo Medical and Dental University, Tokyo, Japan; ${ }^{12}$ The University of Tokyo, Tokyo, Japan; ${ }^{13}$ Hokkaido University, Department of Biostatistics, Sapporo, Japan

Background: Interleukin (IL)-6 pathway inhibitors are suggested by European League Against Rheumatism recommendations to have some advantages in rheumatoid arthritis (RA) patients who are not suitable to use methotrexate as comedication compared with other biological disease-modifying antirheumatic drugs. However, it remains to be elucidated what predicts remission achievement by IL-6 pathway inhibitor monotherapy.

Objectives: To identify predictive factors for clinical and structural remission by tocilizumab without methotrexate in patients with RA.

Methods: This is a post hoc analysis of the SURPRISE study, a 2-year randomized, controlled study comparing the efficacy of tocilizumab with (ADD-ON) and without methotrexate (SWITCH) in active RA patients despite methotrexate therapy. The primary endpoint was the DAS28-ESR remission $(<2.6)$ at week 24 . The change in modified total Sharp score from baseline to week 52 ( $\Delta \mathrm{mTSS} / \mathrm{year}$ ) was also assessed as an endpoint. The effect of clinical parameters, including serum levels of CRP, IL-6, serum amyloid A (SAA), MMP-3, RF and IgG at baseline, on the achievement of DAS28 remission at week 24 was estimated by logistic regression analysis. Multivariate analysis was performed after adjusting for age, gender, height, body weight, body mass index, disease duration, the dose of glucocorticoids, and that of methotrexate.

Results: In SWITCH ( $\mathrm{n}=96)$, CRP, SAA, RF and DAS28 at baseline showed predictive value for DAS28 remission at week 24 in univariate analysis. Multivariate analysis confirmed SAA, RF and DAS28 at baseline, but not CRP, as predictive factors, with SAA having the highest value (OR [95\% Cl] by decrease of $5.0 \mu \mathrm{g} / \mathrm{mL}=1.011$ [1.002-1.020], $\mathrm{p}=0.01$, ROC-AUC $=0.731)$. SAA of $<50.0 \mu \mathrm{g} / \mathrm{mL}$ showed extremely high predictive value for DAS28 remission $(\mathrm{OR}[95 \% \mathrm{Cl}]=6.012[1.997-18.096], \mathrm{p}=$ 0.0008$, ROC-AUC $=0.761)$. Furthermore, structural remission $(\Delta \mathrm{mTSS} /$ year $\leq 0.5)$ rate at week 52 was significantly higher in patients with SAA of $<50.0 \mu \mathrm{g} / \mathrm{mL}$ than those with SAA of $\geq 50.0 \mu \mathrm{g} / \mathrm{mL}(71 \%$ vs $51 \%, p$ $=0.048)$. In contrast, in ADD-ON ( $n=98)$, only DAS28 at baseline showed predictive value for DAS28 remission at week 24 in both univariate and multivariate analysis. In patients with SAA $<50.0 \mu \mathrm{g} / \mathrm{mL}$, both DAS28 remission $(75 \%$ vs $77 \%, p=0.79)$ and structural remission $(71 \%$ vs $68 \%, p=0.71)$ rate were comparable between SWITCH and ADDON.

Conclusion: SAA levels at baseline can predict the necessity of concomitant methotrexate in tocilizumab initiation in patients with RA. Patients with low levels of SAA at baseline may benefit similarly from tocilizumab therapy with and without methotrexate in terms of achieving clinical and structural remission.

\section{REFERENCE:}

[1] Kaneko Y, et al. Ann Rheum Dis. 2016 Nov;75(11):1917-1923. Kaneko Y, et al. Ann Rheum Dis. 2018 Sep;77(9):1268-1275.

Disclosure of Interests: : Masaru Kato Grant/research support from: GSK, Actelion, Speakers bureau: GSK, Actelion, Bayer, Nippon Shinyaku, Eli Lilly, Chugai, Pfizer, Ayumi, Eisai, Asahi Kasei, Yuko Kaneko Grant/ research support from: Abbvie, Eisai, Speakers bureau: AbbVie, Astellas, Ayumi, Bristol-Myers Squibb, Chugai, Eisai, Eli Lilly, Jansen, Kissei, Pfizer, Sanofi, Takeda, Tanabe-Mitsubishi, UCB, Yoshiya Tanaka Grant research support from: Abbvie, Astellas, Bristol-Myers Squibb, Chugai, Daiichi-Sankyo, Eisai, Mitsubishi-Tanabe, MSD, Ono, Taisho-Toyama, Takeda, Speakers bureau: Abbvie, Asahi-kasei, Astellas, Bristol-Myers Squibb, Chugai, Daiichi-Sankyo, Eli Lilly, Eisai, Glaxo-Smithkline, Janssen, Mitsubishi-Tanabe, Novartis, Pfizer Japan Inc, Sanofi, Takeda, UCB, YL Biologics, Masayuki Inoo: None declared, Hitomi Kobayashi-Haraoka: None declared, Koichi Amano Grant/research support from: Asahi Kasei Pharma, Chugai Pharmaceutical Co. Ltd., Speakers bureau: AbbVie GK, Eli Lilly Japan K.K., Mitsubishi Tanabe Pharma, Pfizer Japan Inc., Masayuki Miyata: None declared, Yohko Murakawa Grant/research support from: Asahi Kasei Pharmaceutical Co., Ltd., Chugai Pharmaceutical Co., Ltd., Ono Pharmaceutical Co., Ltd., Daiichi Sankyo Co., Ltd., Teijin Pharma Ltd., Eisai Co., Ltd., Nippon Kayaku Co., Ltd., Mitsubishi Tanabe Pharma Corporation, Paid instructor for: Kissei Pharmaceutical Co., Ltd. Janssen Pharmaceutical K.K. Eisai Co., Ltd., Speakers bureau: Ono Pharmaceutical Co., Ltd., Mitsubishi Tanabe Pharma Corporation, Astellas Pharma Inc., UCB Japan Co. Ltd., Chugai Pharmaceutical Co., Ltd., AbbVie GK, Daiichi Sankyo Co., Ltd., AYUMI Pharmaceutical Corporation, Janssen Pharmaceutical K.K., Takeda Pharmaceutical Company Limited, Sanofi K.K. Teijin Pharma Ltd., Eli Lilly Japan K.K., Eisai Co., Ltd., Hidekata Yasuoka: None declared, Shintaro Hirata Grant/research support from: Eli Lilly, UCB, Consultant for: Bristol-Myers Squibb, Jansen, UCB, Paid instructor for: AbbVie, Eisai, Tanabe-Mitsubishi, Speakers bureau: AbbVie, Astellas, Ayumi, Bristol-Myers Squibb, Chugai, Eisai, Eli Lilly, Jansen, Kissei, Pfizer, Sanofi, Takeda, Tanabe-Mitsubishi, UCB, Hayato Nagasawa: None declared, Eiichi Tanaka Speakers bureau: Abbvie, Asah Kasei pharma co., Bristol Myers Squibb, Chugai Pharmaceutical, Daiichi Sankyo Co., Eisai Pharmaceutical, Janssen Pharmaceutical K.K., Nippon Kayaku, Pfizer, Takeda Pharmaceutical, Taisho Toyama Pharmaceutical Co., and UCB Pharma., Nobuyuki Miyasaka: None declared, Hisashi Yamanaka Grant/research support from: AbbVie, Eisai, Bristol-Meyers, Novartis, Behringer, Astellas, Kaken, Nippon-Shinyaku, Pfizer, UCB, Ayumi, Ono, Daiichi-Sankyo, Taisyo-Toyama, Takeda, Tanabe-Mitsubishi, Chugai, Teijin Pharma, Torii, YLbio, Speakers bureau: Bristol-Meyers, Astellas, Pfizer, Daiichi-Sankyo, Takeda, Tanabe-Mitsubishi, Chugai, Teijin Pharma, YLbio, Kazuhiko Yamamoto Grant/research support from: Astellas, BMS, Daiichi-Sankyo, Mitsubishi Tanabe, Pfizer, Ayumi, Takeda, Chugai, Eisai, Taisho Toyama, UCB, Janssen, Eli Lilly, and NIPPON KAYAKU., Speakers bureau: Astellas, BMS, Daiichi-Sankyo, Mitsubish Tanabe, Pfizer, Ayumi, Takeda, Chugai, Eisai, Taisho Toyama, UCB, Janssen, Eli Lilly, and NIPPON KAYAKU., Isao Yokota Speakers bureau: Chugai Pharma, Tatsuya Atsumi Grant/research support from: Astellas Pharma Inc., Takeda Pharmaceutical Co., Ltd., Mitsubishi Tanabe Pharma Co., Chugai Pharmaceutical Co., Ltd., Daiichi Sankyo Co. Ltd., Otsuka Pharmaceutical Co., Ltd. and Pfizer Inc.. Alexion Inc., Bayer Yakuhin, Ltd,Otsuka Pharmaceutical Co., Ltd. Chugai Pharmaceutical Co., Ltd. Takeda Pharmaceutical Co., Ltd., Eisai Co., Ltd., Bristol-Myers Squibb Co., Daiichi Sankyo Co. Ltd., Mitsubishi Tanabe Pharma Co., Asahi Kasei Pharma Co., Consultant for: ONO PHARMACEUTICAL CO., LTD Sanofi K.K. Daiichi Sankyo Co., Ltd. Pfizer Inc., Speakers bureau: Mitsubishi Tanabe Pharma Co., Chugai Pharmaceutical Co., Ltd., Astellas Pharma Inc., Takeda Pharmaceutical Co., Ltd., Pfizer Inc.,Daiichi Sankyo Co. Ltd., Bristol-Myers Squibb Co., Eli Lilly Japan K.K., Tsutomu Takeuchi Grant/ research support from: Astellas Pharma Inc, Chugai Pharmaceutical Co, Ltd., Daiichi Sankyo Co., Ltd., Takeda Pharmaceutical Co., Ltd., AbbVie GK, Asahikasei Pharma Corp., Mitsubishi Tanabe Pharma Co., Pfizer Japan Inc., Eisai Co., Ltd., AYUMI Pharmaceutical Corporation, Nipponkayaku Co. Ltd., Novartis Pharma K.K., Grant/research support from: AbbVie, Asahi Kasei, Astellas, AstraZeneca, AYUMI, Bristol-Myers Squibb, Chugai, Daiichi Sankyo, Eisai, Eli Lilly Japan, Janssen, Mitsubishi Tanabe, Nippon Kayaku, Novartis, Pfizer Japan Inc, Taiho, Taisho Toyama, Takeda, Teijin, Grant/research support from: Astellas Pharma Inc., Bristol Myers Squibb, Chugai Pharmaceutical Co., Ltd., Mitsubishi Tanabe Pharma Co., Pfizer Japan Inc., Santen Pharmaceutical Co., Ltd. Takeda Pharmaceutical Co., Ltd., Teijin Pharma Ltd., AbbVie GK, Asahi 
Kasei Pharma Corp., Taisho Toyama Pharmaceutical Co., Ltd., SymBio Pharmaceuticals Ltd., Janssen Pharmaceutical K.K., Celltrion Inc., Nipponkayaku Co. Ltd., and UCB Japan, Consultant for: Astra Zeneca K.K., Eli Lilly Japan K.K., Novartis Pharma K.K., Mitsubishi Tanabe Pharma Co., Abbivie GK, Nipponkayaku Co.Ltd, Janssen Pharmaceutical K.K., Astellas Pharma Inc., Taiho Pharmaceutical Co. Ltd., Chugai Pharmaceutical Co. Ltd., Taisho Toyama Pharmaceutical Co. Ltd., GlaxoSmithKline K.K., UCB Japan Co. Ltd., Consultant for: AbbVie, Asahi Kasei, Astellas, AstraZeneca, AYUMI, Bristol-Myers Squibb, Chugai, Daiichi Sankyo, Eisai, Eli Lilly Japan, Janssen, Mitsubishi Tanabe, Nippon Kayaku, Novartis, Pfizer Japan Inc, Taiho, Taisho Toyama, Takeda, Teijin, Consultant for: Astra Zeneca K.K., Eli Lilly Japan K.K., Novartis Pharma K.K., Mitsubishi Tanabe Pharma Co., Asahi Kasei Medical K.K., AbbVie GK, Daiichi Sankyo Co., Ltd., Bristol Myers Squibb, and Nipponkayaku Co. Ltd., Speakers bureau: Astellas Pharma Inc., Bristol Myers Squibb, Chugai Pharmaceutical Co., Ltd., Mitsubishi Tanabe Pharma Co., Pfizer Japan Inc., Santen Pharmaceutical Co., Ltd., Takeda Pharmaceutical Co., Ltd., Teijin Pharma Ltd., AbbVie GK, Asahi Kasei Pharma Corp., Taisho Toyama Pharmaceutical Co., Ltd., SymBio Pharmaceuticals Ltd., Janssen Pharmaceutical K.K., Celltrion Inc., Nipponkayaku Co. Ltd., and UCB Japan, Speakers bureau: AbbVie, Asahi Kasei, Astellas, AstraZeneca, AYUMI, Bristol-Myers Squibb, Chugai, Daiichi Sankyo, Eisai, Eli Lilly Japan, Janssen, Mitsubishi Tanabe, Nippon Kayaku, Novartis, Pfizer Japan Inc, Taiho, Taisho Toyama, Takeda, Teijin, Speakers bureau: AbbVie GK., Bristol-Myers K.K., Chugai Pharmaceutical Co. Ltd., Mitsubishi Tanabe Pharma Co., Pfizer Japan Inc., Astellas Pharma Inc, Diaichi Sankyo Co. Ltd., Eisai Co. Ltd., Sanofi K.K., Teijin Pharma Ltd., Takeda Pharmaceutical Co. Ltd., Novartis Pharma K.K.

DOI: 10.1136/annrheumdis-2019-eular.505

\section{FRI0100 TOWARDS THE LOWEST EFFICACIOUS DOSE (TOLEDO): RESULTS OF A MULTICENTER NON- INFERIORITY RANDOMIZED OPEN-LABEL CONTROLLED TRIAL ASSESSING TOCILIZUMAB OR ABATACEPT INJECTION SPACING IN RHEUMATOID ARTHRITIS PATIENTS IN REMISSION}

KEDRA Joanna, Philippe Dieudé, Hubert Marotte, Alexandre Lafourcade, Emilie Ducourau, Thierry Schaeverbeke, Aleth Perdriger, Martin Soubrier, Jacques Morel, Arnaud Constantin, Emmanuelle Dernis, Valérie Royant, JeanHugues Salmon, Thao Pham, Jacques-Eric Gottenberg, Edouard Pertuiset, Maxime Dougados, Valerie Devauchelle-Pensec, Philippe Gaudin,

Gregoire Cormier, Philippe Goupille, Xavier Mariette, Francis Berenbaum, Didier Alcaix, Sid Ahmed Rouidi, Jean-Marie Berthelot, Agnès Monnier, Christine Piroth, Frederic Lioté, Vincent Goeb, Cecile Gaujoux-Viala, Isabelle Chary Valckenaere, David Hajage, Florence Tubach, Bruno Fautrel. CRI-IMIDIATE clinical research network, Paris, France

Background: Biologic Disease Modifying Anti-Rheumatic Drugs (bDMARD) tapering is possible in rheumatoid arthritis (RA) patients in sustained remission. However, only minimal data are available on progressive tapering of non-TNF bDMARD such as tocilizumab (TCZ) or abatacept (ABA). Objectives: The TOLEDO (Towards the Lowest Efficacious Dose) trial aimed to assess the impact on disease activity of progressive spacing of TCZ or ABA in RA patients in sustained remission compared to their maintenance at full dose.

Methods: In this multicenter open-label non-inferiority randomized controlled trial, patients fulfilling ACR-EULAR 2010 criteria for RA were included if they were 1) treated with $\mathrm{ABA}$ or TCZ for $\geq 1$ year (monotherapy or in combination with csDMARD, corticosteroid allowed at a dose $\leq 5 \mathrm{mg} /$ day), 2) in DAS28VS remission (DAS28 <2.6) for $\geq 6$ months and 3) with no X-ray damage progression in the year before inclusion. They were randomized into 2 arms: TCZ or ABA maintenance at full dose or DAS28-driven progressive injection spacing arm adapted in which bDMARD IV or SC injections were progressively spaced out every 3 months according to a predetermined 4-step algorithm up to bDMARD discontinuation at step 4. Spacing was reversed to the previous interval in case of relapse. The primary outcome was the evolution of disease activity according to DAS44 during the 2-year follow-up, which was analyzed with a linear mixed-effect model. Relapse and durable relapse rates (respectively defined as DAS28 > 3.2, and DAS28 >3.2 not recovered at the following visit despite bDMARD escalation at previous step) were also compared between the 2 arms. Analysis were done per protocol (PP) according to a non-inferiority hypothesis (non-inferiority margin at 0.25 for DAS44 and 0.07 for relapse rates).

Results: 117 patients were randomized in Spacing arm and 116 in Maintenance $\operatorname{arm}(90$ and 112 respectively for PP analysis). 165 (72.4\%) patients were treated with TCZ and 63 (27.6\%) with ABA. At the end of the follow-up in the Spacing arm, $12.4 \%$ of patients were able to discontinue their bDMARD (step 4),38.9\% had tapered them (step 1 to 3 ) and $23.9 \%$ needed to go back to initial step (step 0). In terms of disease activity, the non-inferiority of the Spacing strategy in terms of disease activity (DAS44) was not demonstrated for the whole population and the ABA subgroups: slope difference of $11 \%(95 \% \mathrm{Cl}:-9 \%, 32 \%)$ and $37 \%$ (95\% Cl: $-4 \%, 77 \%)$ respectively. However, it was satisfied for the TCZ subgroup: slope difference $3 \%(95 \% \mathrm{Cl}:-21 \%, 27 \%)$ (Figure 1). Relapses (Figure 2) were more frequent in the Spacing arm: $+45 \%(95 \% \mathrm{Cl}: 32 \%$, $57 \%),+48 \%(95 \% \mathrm{Cl}: 24 \%, 71 \%)$ and $+43 \%(95 \% \mathrm{Cl}: 29 \%, 58 \%)$ in the whole population, $\mathrm{ABA}$ and TCZ subgroups respectively. Durable relapses were more frequent in the Spacing arm: $+10 \%(95 \% \mathrm{Cl}: 0 \%, 19 \%), 16 \%$ $(95 \% \mathrm{Cl}:-5 \%, 37 \%)$ and $7 \%(95 \% \mathrm{Cl}:-3 \%, 16 \%)$ in the whole population, ABA and TCZ subgroups respectively, compared with Maintenance arm.

Conclusion: The TOLEDO trial generally failed to demonstrate the noninferiority of the proposed tapering strategy in comparison to maintenance at full dose. However, the non-inferiority was satisfied in terms of disease activity for the TCZ subgroup.

Disclosure of Interests: Joanna KEDRA: None declared, Philippe Dieudé: None declared, Hubert MAROTTE: None declared, Alexandre Lafourcade: None declared, Emilie Ducourau Speakers bureau: BMS and Abbvie Thierry Schaeverbeke: None declared, Aleth Perdriger: None declared Martin SOUBRIER: None declared, Jacques Morel: None declared, Arnaud Constantin: None declared, Emmanuelle Dernis: None declared Valérie Royant: None declared, Jean-Hugues Salmon Speakers bureau: Janssen Novartis, Thao Pham Speakers bureau: Lilly, Novartis, JacquesEric Gottenberg Grant/research support from: Bristol-Myers Squibb, Grant research support from: Bristol-Myers Squibb, Consultant for: Bristol-Myers Squibb, Lilly, Pfizer, Sanofi-Genzyme, UCB Pharma, Consultant for: Bristol-Myers Squibb, Eli Lilly, UCB, Sanofi-Genzyme, Pfizer, Edouard Pertuiset: None declared, maxime dougados Grant/research support from: El Lilly and Company, Pfizer, AbbVie, and UCB Pharma, Consultant for: Eli Lilly and Company, Pfizer, AbbVie, and UCB Pharma, Valerie Devau chelle-Pensec Grant/research support from: Roche-Chugai, Speakers bureau: MSD, BMS, UCB, Roche, Philippe Gaudin Speakers bureau: Roche, Chugai, BMS, Abbvie, Servier, Pfizer, MSD, UCB, ESAOTE, Genévrier, Janssen, Novartis, Lilly, Biogen, Amge, Gregoire CORMIER: None declared, Philippe Goupille: None declared, Xavier Mariette Grant/research support from: Servier, Consultant for: AstraZeneca, Bristol-Myers Squibb, GlaxoSmithKline, Janssen, Pfizer, UCB Pharma, Francis Berenbaum None declared, Didier Alcaix: None declared, SID AHMED ROUIDI: None declared, Jean-Marie Berthelot: None declared, Agnès Monnier: None declared, Christine Piroth: None declared, Frederic Lioté Grant/research support from: institutional grants from Grunenthal, Ipsen Pharma/Menarini, Novartis, SOBI for the European Crystal Network Workshops, Consultant for: Grunenthal, Novartis, Vincent Goeb: None declared, Cecile Gaujoux Viala Consultant for: Speaking and/or consulting fees from AbbVie, Amgen, Bristol-Myers Squibb, Celgene, Eli Lilly, Gilead, Janssen, MerckSerono, Medac, Nordic Pharma, Novartis, Pfizer, Roche, Sandoz, Sanofi and UCB Pharma., Speakers bureau: Speaking and/or consulting fees from AbbVie, Amgen, Bristol-Myers Squibb, Celgene, Eli Lilly, Gilead, Janssen, Merck-Serono, Medac, Nordic Pharma, Novartis, Pfizer, Roche, Sandoz, Sanofi and UCB Pharma., Isabelle CHARY VALCKENAERE: None declared, David Hajage: None declared, Florence Tubach Grant/ research support from: Financial compensation received from MSD on a pro-rota basis for participation in Scientific Committee meetings and functions for this study, Bruno Fautrel Grant/research support from: AbbVie, Lilly, MSD, Pfizer, Consultant for: AbbVie, Biogen, BMS, Celgene, Janssen, Lilly, Medac, MSD, NORDIC Pharma, Novartis, Pfizer, Roche, Sanofi-Aventis, Sanofi Genzyme, SOBI, UCB 